\title{
COMPLEX AND DISTRIBUTIONAL WEIGHTS FOR \\ SIEVED ULTRASPHERICAL POLYNOMIALS
}

\author{
JAIRO A. CHARRIS \\ Departamento de Matemáticas y Estadística \\ Universidad Nacional de Colombia \\ Bogotá, COLOMBIA \\ FELIX H. SORIANO \\ Departamento de Matemáticas y Estadística \\ Universidad Nacional de Colombia \\ Universidad de los Andes \\ Bogotá, COLOMBIA \\ (Received November 11, 1992 and in revised form May 5, 1993)

\begin{abstract}
Contour integral and distributional orthogonality of sieved ultraspherical polynomials are established for values of the parameters outside the natural range of orthogonality by positive measures on the real line. A general representation theorem for moment functionals is included.
\end{abstract}

KEY WORDS AND PHRASES. Ultraspherical and sieved ultraspherical polynomials, Jacobi polynomials, Chebyshev polynomials, recurrence relations, continued fractions, orthogonality measures, distributions, contour integrals, moment functionals.

1992 AMS SUBJECT CLASSIFICATION CODES. Primary 33A65. Secondary 33A30, 42C05

\section{INTRODUCTION AND PRELIMINARIES.}

A (normalized) moment functional $\mathcal{L}$ (Ahkiezer [1], Chihara [9], Shohat and Tamarkin [17], Stone [18]) is a complex linear map of the space $\mathbb{C}[x]$ of complex polynomials into the field $\mathbb{C}$ of complex numbers such that $\mathcal{L}(1)=1$. A sequence $\left\{P_{n}(x): n \geq 0\right\}$ of polynomials in $\mathbb{C}[x]$ with $P_{n}(x)$ of degree $n$ and $P_{0}(x)=1$ is orthogonal with respect to $\mathcal{L}$ if

$$
\mathcal{L}\left(P_{n}(x) P_{m}(x)\right)=\lambda_{n} \delta_{m n}, m, n \geq 0,
$$

with

$$
\lambda_{0}=1 ; \lambda_{n} \neq 0, n>0 .
$$

The functional $\mathcal{L}$ is also called an orthogonality functional for $\left\{P_{n}(x)\right\}$. It is well known (Chihara [9], Chaps. I, II) that $\left\{P_{n}(x)\right\}$ is orthogonal with respect to $\mathcal{L}$ if and only if there are numbers $A_{n}, B_{n}, C_{n}$ such that

$$
A_{n} C_{n+1} \neq 0, n \geq 0
$$

and

$$
x P_{n}(x)=A_{n} P_{n+1}(x)+B_{n} P_{n}(x)+C_{n} P_{n-1}(x), n \geq 0,
$$


with $P_{-1}(. x)=0 . P_{0}(x)=1$. Furthermore. $\left\{P_{n}(x)\right\}$ determines $\mathcal{L}$ uniquely by

$$
\mathcal{L}(1)=1: \mathcal{L}\left(P_{n}(x)\right)=0 ., " \geq 1 \text {. }
$$

and the 1elationship

$$
\lambda_{n}=\mathcal{L}\left(P_{n}^{2}(x)\right)=\frac{C_{1} \cdots C_{n}}{A_{1} \quad A_{n-1}} \cdot n \geq 1
$$

holds.

The functional $\mathcal{L}$ can be represented by means of a positive measure $\mu$ supported by the real line. i.e.

$$
\mathcal{L}(P(x))=\int_{-\infty}^{+\infty} P(x) d \mu(x),
$$

if and only if $A_{n}, B_{n}, C_{n}$ are real numbers and

$$
A_{n} C_{n+1}>0, n \geq 0 .
$$

This is known as Favard's theorem (Chihara [9], Chap. II). The measure $\mu$ is called an orthogonality or a spectral measure for $\left\{P_{n}(x)\right\}$, and $\left\{P_{n}(x)\right\}$ is said to be orthogonal with respect to $\mu$.

For most systems of polynomials defined by a recurrence relation (1.4), such as those of Laguerre and Jacobi, the recurrence coefficients depend on certain numerical parameters $\alpha, \beta, \lambda, \ldots$, and (1.8) holds for these parameters within certain ranges (called their natural ranges), but breaks down for most values of them. However, (1.3) usually holds for all $\alpha, \beta, \lambda, \ldots$, except, perhaps, for countably many.

This has motivated the search for other types of representations of $\mathcal{L}$. (Krall [14] and Morton and Krall [15]) have discussed representations of $\mathcal{L}$ in terms of distributions supported by the real line for several classical systems. Recently, Ismail, et al. [13] have given representations of $\mathcal{L}$ for some systems of polynomials in terms of measures supported by curves of the complex plane and have used those representations to derive distributional representations of $\mathcal{L}$. They prove in each case that

$$
\mathcal{L}(P(x))=\frac{1}{2 \pi i} \int_{C} P(z) X(z) d z
$$

where $C$ is a positively oriented closed contour of the plane contained in the domain of analiticity of $X(z)$, the limit function of the continued fraction

$$
\frac{1 \mid}{\mid z-B_{0}}-\frac{A_{0} C_{1} \mid}{\mid z-B_{1}}-\frac{A_{1} C_{2} \mid}{\mid z-B_{2}}-
$$

of the polynomials $\left\{P_{n}(x)\right\}$, is a representation of $\mathcal{L}$.. For example, for the ultraspherical polynomials $\left\{C_{n}^{\lambda}(x)\right\}$, the special case $\alpha=\beta=\lambda-1 / 2$ of the Jacobi polynomials, the recurrence coefficients are (Rainville [16], Chap. 17)

$$
A_{n}=\frac{n+1}{2(n+\lambda)}, B_{n}=0, C_{n}=\frac{n+2 \lambda-1}{2(n+\lambda)}, n \geq 0,
$$

and (1.8) holds if and only if $(n+\lambda)(n+\lambda+1)(n+2 \lambda)>0, n \geq 0$, which amounts to $\lambda \neq$ $0, \lambda>-1 / 2$. However, (1.3) holds as long as $2 \lambda \neq 0$ and is not a negative integer. Under this assumption, the continued fraction of $\left\{C_{n}^{\lambda}(x)\right\}$ converges to

$$
X^{\lambda}(z)=\frac{1}{z \pm 1}{ }_{2} F_{1}\left(\begin{array}{c|c}
1, \lambda+\frac{1}{2} & \frac{2}{2 \lambda+1} \\
1 \pm z
\end{array}\right)
$$


provided that $|z \pm 1|>2$. Here ${ }_{2} F_{1}$ stands for the hypergeometrie series (Rainville [16]. Chap. 4)

$$
{ }_{2} F_{1}\left(\begin{array}{cc|c}
a & b & \xi \\
c & & \xi
\end{array}\right)=\sum_{n=0}^{\infty} \frac{(a)_{n}(b)_{n}}{n !(c)_{n}} \xi^{n}, \quad|\xi|<1 .
$$

where $(a)_{0}=1 .(a)_{n}=a(a+1) \ldots(a+n-1), n \geq 1$ (so that $c$ in $(1.13)$ can not be zero nor a negative integer). On the other hand (Rainville [16]. p. 279 )

$$
C_{m}^{\lambda}(x)=\sum_{k=0}^{m} \frac{(2 \lambda)_{m+k}(x-1)^{k}}{2^{k} k !(m-k) !(\lambda+1 / 2)_{k}}, m \geq 0
$$

Hence, if $\mathcal{L}_{0}$ is defined by (1.9) with $X(z)=X^{\lambda}(z)$ and $C$ a positively oriented closed contour in $|z \pm 1|>2$ containing \pm 1 in its interior, a simple calculation, which takes into account that $(2 \lambda)_{m+n}=(2 \lambda)_{m}(2 \lambda+m)_{n}$ and $(-1)^{n}(-m)_{n}=m ! /(m-n) !$, shows that

$$
\mathcal{L}_{0}\left(C_{m}^{\lambda}(x)\right)=\frac{(2 \lambda)_{m}}{m !}{ }_{2} F_{1}\left(\begin{array}{cc|}
-m, & 2 \lambda+m \\
2 \lambda+1 & 1
\end{array}\right)
$$

and the $\mathrm{Chu}$-Vandermonde sum

$$
{ }_{2} F_{1}\left(\begin{array}{cc|c}
-m, & b & 1 \\
c & & 1
\end{array}\right)=\frac{(c-b)_{m}}{(c)_{m}}
$$

(Rainville [16], p. 69) then implies that $\mathcal{L}_{0}(1)=1, \mathcal{L}_{0}\left(C_{m}^{\lambda}(x)\right)=0, m>0$. Hence, $\mathcal{L}_{0}$ is, in view of (1.5), the orthogonality functional of $\left\{C_{n}^{\lambda}(x)\right\}$.

In section 2 we will prove that representation (1.9) responds to a general situation, implying that a check up as above is unnecessary. Now we derive from (1.9) a distributional representation of $\mathcal{L}_{0}$. For $|z \pm 1|>2$,

$$
X^{\alpha, \beta}(z)=2^{\alpha+\beta+1} \frac{\Gamma(\alpha+1) \Gamma(\beta+1)}{\Gamma(\alpha+\beta+2)} \frac{1}{z-1}{ }_{2} F_{1}\left(\begin{array}{c|c}
1, & \alpha+1 \\
\alpha+\beta+2 & \frac{2}{1-z}
\end{array}\right)
$$

is the continued fraction limit function of the system $\left\{P_{n}^{(\alpha, \beta)}(x)\right\}$ of Jacobi polynomials (Szegö [19], §4.61). Then (Szegö [19], (4.1.4)) $X^{\alpha, \beta}(-z)=-X^{\beta, \alpha}(z)$ and

$$
\begin{aligned}
& \int_{C} P(z)(1-z)^{m} X^{\alpha, \beta}(z) d z=\int_{C} P(z) X^{\alpha+m, \beta}(z) d z \\
& \int_{C} P(z)(1+z)^{m} X^{\alpha, \beta}(z) d z=\int_{C} P(z) X^{\alpha, \beta+m}(z) d z
\end{aligned}
$$

for any polynomial $P(z)$, any positively oriented contour in $|z \pm 1|>2$ containing [-1,1] in its interior, and any integer $m \geq 0$. Thus,

$$
\int_{C} P(z)\left(1-z^{2}\right)^{m} X^{\alpha, \beta}(z) d z=\int_{C} P(z) X^{\alpha+m, \beta+m}(z) d z
$$

Recalling that $(\alpha)_{n}=\Gamma(\alpha+n) / \Gamma(\alpha)$, and Legendre's duplication formula (Rainville [16], p. 24), we see that

$$
X^{\lambda}(z)=\frac{\Gamma(\lambda+1)}{\sqrt{\pi} \Gamma(\lambda+1 / 2)} X^{\lambda-1 / 2, \lambda-1 / 2}(z),|z \pm 1|>2 .
$$

Since $\varphi(x)(1-x)^{\alpha}(1+x)^{\beta} d x$, where $\varphi$ is the characteristic function of $[-1,1]$, is, provided $\alpha, \beta>-1$, the orthogonality measure of $\left\{P_{n}^{(\alpha, \beta)}(x)\right\}$ (Rainville [16], p. 258; Szegö [19], §4.3), then

$$
\frac{1}{2 \pi \imath} \int_{C} P(z) X^{\alpha, \beta}(z) d z=\int_{-1}^{1} P(x)(1-x)^{\alpha}(1+x)^{\beta} d x .
$$


In particular.

$$
\mathcal{L}_{0}(P(x))=\frac{\Gamma(\lambda+1)}{\sqrt{\pi} \Gamma(\lambda+1 / 2)} \int_{-1}^{1} P(x)\left(1-x^{2}\right)^{\lambda-1 / 2} d x, \quad \lambda>-1 / 2 .
$$

Now. for $P(x) \in \mathbb{C}[x]$ and $m \geq 0$ an integer. we have

$$
\frac{P(x)}{\left(1-x^{2}\right)^{m}}=\sum_{i=0}^{1} \sum_{\jmath=1}^{m} \frac{\alpha_{\imath \jmath}}{\left(x-\xi_{\imath}\right)^{\jmath}}+R(x) ; \quad \alpha_{\imath \jmath}=\frac{(-1)^{m}}{(m-\jmath) !} \frac{d^{m-\jmath}}{d x^{m-\jmath}}\left[\frac{P(x)}{\left(x+\xi_{\imath}\right)^{m}}\right]\left(\xi_{\imath}\right)
$$

where $\xi_{0}=-1, \xi_{1}=1$ and $R(x)$ is a polynomial, and ( 115$),\left(\begin{array}{lll}1 & 17\end{array}\right)$ then yield

$$
\begin{aligned}
\mathcal{L}_{0}(P(x))= & 2^{m} \frac{(\lambda+1 / 2)_{m}}{(2 \lambda+1)_{m}} \sum_{\imath=0}^{1} \sum_{\jmath=0}^{m-1} \frac{(-2)^{\jmath} \xi_{\imath}^{m+\jmath}}{\jmath !} \cdot \frac{(\lambda+1 / 2)_{\jmath}}{(2 \lambda+m+1)_{\jmath}} \cdot \frac{d^{\jmath}}{d x^{\jmath}}\left[\frac{P(x)}{\left(x+\xi_{\imath}\right)^{m}}\right]\left(\xi_{\iota}\right)+ \\
& +\frac{\Gamma(\lambda+1)}{\sqrt{\pi} \Gamma(\lambda+1 / 2)} \int_{C} R(z) X^{\lambda+m-1 / 2, \lambda+m-1 / 2}(z) d z .
\end{aligned}
$$

THEOREM 1. Provided $2 \lambda \neq 0$ is not a negative integer and $\lambda>-m-1 / 2$, where $m \geq 0$ is an integer, we have the distributional representation $\mathcal{L}_{0}=T_{01}+T_{02}$ where, for any test function $\varphi$,

$$
T_{01}(\varphi)=2^{m} \frac{(\lambda+1 / 2)_{m}}{(2 \lambda+1)_{m}} \sum_{\imath=0}^{1} \sum_{\jmath=0}^{m-1} \frac{(-2)^{\jmath} \xi_{\imath}^{m+\jmath}}{j !} \frac{(\lambda+1 / 2)_{\jmath}}{(2 \lambda+m+1)_{\jmath}} \frac{d^{\jmath}}{d x^{\jmath}}\left[\frac{P(x)}{\left(x+\xi_{\imath}\right)^{m}}\right]\left(\xi_{\imath}\right)
$$

and

$$
T_{02}(\varphi)=\frac{\Gamma(\lambda+1)}{\sqrt{\pi} \Gamma(\lambda+1 / 2)} \int_{-1}^{1} \varphi_{m}(x)\left(1-x^{2}\right)^{\lambda+m-1 / 2} d x,
$$

with $\xi_{0}=-1, \xi_{1}=1$ and

$$
\varphi_{m}(x)=\frac{\varphi(x)}{\left(1-x^{2}\right)^{m}}-\sum_{\imath=0}^{1} \sum_{\jmath=0}^{m-1} \frac{(-1)^{m}}{\jmath !} \frac{d^{\jmath}}{d x^{\jmath}}\left[\frac{P(x)}{\left(x+\xi_{\imath}\right)^{m}}\right]\left(\xi_{\imath}\right) \frac{1}{\left(x-\xi_{\imath}\right)^{m-\jmath}}
$$

The distributions $T_{01}$ and $T_{02}$ have compact support on the real line and can act on polynomials.

We will show in this work that representation

$$
\mathcal{L}_{0}(P(x))=\frac{1}{2 \pi i} \int_{C} P(z) X^{\lambda}(z) d z
$$

of $\mathcal{L}_{0}$ can be "lifted" to contour integral representations of the orthogonality functionals $\mathcal{L}_{1}$ and $\mathcal{L}_{2}$ of the sieved ultraspherical polynomials of the first and second kinds. Then, from such representations, we will derive distributional representations of $\mathcal{L}_{1}$ and $\mathcal{L}_{2}$

The monic sieved ultraspherical polynomials of the first kind, $\left\{p_{n}^{\lambda}(x)\right\}$, and of the second kind, $\left\{q_{n}^{\lambda}(x)\right\}$, satisfy the blocks of recurrence relations

$$
x y_{n k+\jmath}=y_{n k+\jmath+1}+a_{n}^{(\jmath)} y_{n k+\jmath-1}, n \geq 0, \quad j=0,1,2, \ldots, k-1,
$$

with $y_{-1}=0, y_{0}=1$. The coefficients $a_{n}^{(\jmath)}$ are

$$
a_{n}^{(0)}=\frac{n}{4(n+\lambda)}, a_{n}^{(1)}=\frac{n+2 \lambda}{4(n+\lambda)}, a_{n}^{(3)}=\frac{1}{4}, \quad j=2,3, \ldots, k-1, n \geq 0,
$$

for $\left\{p_{n}^{\lambda}(x)\right\}$, and

$$
a_{n}^{(0)}=\frac{n}{4(n+\lambda)}, a_{n}^{(k-1)}=\frac{n+2 \lambda+1}{4(n+\lambda+1)}, a_{n}^{(\jmath)}=\frac{1}{4}, \quad \jmath=1,2, \ldots, k-2, n \geq 0,
$$


for $\left\{q_{n}^{\lambda}(. r)\right\}$. It is assumed that $k \geq 2$ is an integer.

Siered ultraspherieal polynomials were introduced by Al-Salam et al. [2]. Generalizations and interpretations of the sieving process are in Charris and Ismail [5 7], Charris ot al [8]. while Charris and Ismail [6]. Ismail [11 12] contain specific examples of sieved polynomial systems. Geronimo and Van Assche [10] also present an interesting approach to sieved orthogonal polynomials.

The paper iñ organized as follows: In Section 2 we prove a general result about sepresentations (1.9). and some lemmas, related to the Chebysher polynomials, which will be necded in the sequel. Sections 3 and 4 contain contour integral representations of $\mathcal{L}_{1}$ and $\mathcal{L}_{2}$, and Sectum 5 . their distributional representations.

In closing this section we observe that since $B_{n}=0$ for all $n \geq 0$ in (1.11), then

$$
C_{n}^{\lambda}(-x)=(-1)^{n} C_{n}^{\lambda}(x), n \geq 0 .
$$

It is convenicut to assume that $C_{-1}^{\lambda}(x)=0$. Now let

$$
C_{n-1}^{\lambda}(z ; 1)=\frac{1}{2 \lambda} \mathcal{L}_{0}\left(\frac{C_{n}^{\lambda}(x)-C_{n}^{\lambda}(z)}{x-z}\right), n \geq 0 .
$$

Then $\left\{C_{n}^{\lambda}(x ; 1)\right\}$ is a system of polynomials (Chihara [9], Chap. III), called the numerator polynomials of $\left\{C_{n}^{\lambda}(x)\right\}$. Since

$$
\int_{C} \frac{X^{\lambda}(z)}{z-1} d z=\int_{C} \frac{X^{\lambda}(z)}{z+1} d z=0
$$

for any closed contour of $|z \pm 1|>2$ containing \pm 1 in its interior, as follows from (1.12), then

$$
2 \lambda C_{n}^{\lambda}( \pm 1 ; 1)=\frac{1}{2 \pi \imath} \int_{C} \frac{C_{n+1}^{\lambda}(z)}{z \mp 1} X^{\lambda}(z) d z, n \geq 0
$$

Relation (1.23) also implies that

$$
C_{n}^{\lambda}(-x ; 1)=(-1)^{n} C_{n}^{\lambda}(x ; 1), n \geq 0 .
$$

\section{BASIC RESULTS.}

Theorem 26.2, p. 112, of Wall [20] ensures that (1.10) is uniformly convergent on $|z| \geq M^{\prime}$ for all $M^{\prime}>M$, provided that (1.3) and

$$
\left|B_{n}\right| \leq M / 3,\left|A_{n} C_{n+1}\right| \leq M^{2} / 9, n=0,1,2, \ldots
$$

hold.

Hence, $X(z)$ is analytic in $|z|>M$. We claim that

THEOREM 2. If $\left\{P_{n}(x)\right\}$ is determined by (1.4), and (1.3), (2.1) hold, the moment functional $\mathcal{L}$ of $\left\{P_{n}(x)\right\}$ is represented by (1.9) for any positively oriented closed contour $C$ of $|z|>M$ containing 0 in its interior.

PROOF. Because of the uniform convergence to $X(z)$ of (1.10), we may assume that the zeros of all the $P_{n}(x)$ 's are interior to $C$. From the general theory of continued fractions (Askey and Ismail [3], Chihara [9], Wall [20]) it follows that

$$
X(z)=\lim _{n \rightarrow \infty} \frac{P_{n}^{*}(z)}{P_{n}(z)},|z|>M,
$$

where $\left\{P_{n}^{*}(x)\right\}$ is the system of polynomials determined by (1.4) for $n \geq 1$ and $P_{0}^{*}(x)=0, P_{1}^{*}(x)=$ $1 / A_{0}$. Induction on (1.4) readily shows that

$$
P_{n+1}^{*}(x) P_{n}(x)-P_{n+1}(x) P_{n}^{*}(x)=\frac{\lambda_{n}}{A_{n}}, n \geq 0,
$$


where $\lambda_{n}$ is given by (1.6). Relation (2.3) is known as Abel's formula (Ahkiezer [1], Chihara [9]). Since

$$
\frac{1}{2 \pi \imath} \int_{C} \frac{P_{n}^{*}(z)}{P_{n}(z)} d z=1, n \geq 1
$$

and for $m \geq 1$

$$
\begin{aligned}
\int_{C} P_{n}(z) \frac{P_{n+m}^{*}(z)}{P_{n+m}(z)} d z & =\sum_{k=1}^{m} \int_{C} P_{n}(z)\left[\frac{P_{n+k}^{*}(z)}{P_{n+k}(z)}-\frac{P_{n+k-1}^{*}(z)}{P_{n+k-1}(z)}\right] d z+\int_{C} P_{n}^{*}(z) d z \\
& =\sum_{k=1}^{m} \frac{\lambda_{n+k-1}}{A_{n+k-1}} \int_{C} \frac{P_{n}(z)}{P_{n+k}(z) P_{n+k-1}(z)} d z \\
& =0
\end{aligned}
$$

then

$$
\frac{1}{2 \pi \imath} \int_{C} X(z) d z=1 ; \frac{1}{2 \pi \imath} \int_{C} P_{n}(z) X(z) d z=0, n \geq 1,
$$

and the assertion follows.

For $|z|>M$ we have the Laurent expansion (see Wall [20], pp. 192-211)

$$
X(z)=\sum_{n=0}^{\infty} \frac{u_{n}}{z^{n+1}}
$$

where

$$
u_{0}=1 ; u_{n}=\frac{1}{2 \pi i} \int_{C} z^{n} X(z) d z, \quad n \geq 0
$$

Hence,

$$
\lim _{z \rightarrow \infty} z X(z)=1
$$

We have

THEOREM 3. Assume (1.3) and (2.1) hold, and that the moment functional $\mathcal{L}$ of $\left\{P_{n}(x)\right\}$ has the representation

$$
\mathcal{L}(P(x))=\frac{1}{2 \pi i} \int_{C} P(z) Y(z) d z
$$

where $Y(z)$ is analytic on $|z|>M, \lim _{z \rightarrow \infty} Y(z)=0$, and $C$ is a positively oriented closed contour in $|z|>M$ containing 0 in its interior. Then $Y(z)=X(z)$ for all $|z|>M$.

PROOF. Since

$$
\int_{C} P_{n}(z)(X(z)-Y(z)) d z=0, n \geq 0,
$$

and $\left\{P_{n}(x)\right\}$ is an algebraic basis of $\mathbb{C}[x]$, also

$$
\int_{C} z^{n}(X(z)-Y(z)) d z=0, n \geq 0
$$

Thus, if

$$
X(z)-Y(z)=\sum_{n=-\infty}^{+\infty} a_{n} z^{n},|z|>M
$$

is the Laurent expansion of $X(z)-Y(z)$, then

$$
a_{-n-1}=\frac{1}{2 \pi i} \int_{C} z^{n}(X(z)-Y(z)) d z=0, n \geq 0,
$$

and $X(x)-Y(z)$ is an entire function vanishing at $\infty$. Hence $X(z)=Y(z)$ for $|z|>M$. 
The Chebraher polynomals of the first kind. $\left\{T_{n}(r)\right\}$. and of the second kind. $\left\{U^{*}(r)\right\}$. both satisfy the recurrence relation $2 . r y_{n}=y_{n+1}+y_{n-1}, n \geq 1$. but $T_{n}(x)=1 . T_{1}(x)=x$ while $L_{01}(x)=1 . C_{1}(x)=2 r$. We alos assume that $T_{-1}(x)=U_{-1}^{r}(x)=0$.

For 1 an integer. let cos, be the restriction map of $\cos$ to $\Omega_{t}=(1 \pi \cdot(1+1) \pi) \times \mathbb{R}$. where $\mathbb{R}$ denotes the real line. If $\Omega$ is the complex plane cut along $(-\infty,-1]$ and $[-1++\infty)$. cos, maps $\Omega_{1}$ conformally onto $\Omega$. Whe denote with coss ${ }_{2}^{-1}$ the analytic inverse of con, on $\Omega$. Clearly

$$
T_{k}(z)=\cos \left(k \cdot \cos _{0}^{-1}(z)\right) \cdot U_{k}(z)=\frac{\sin \left((k+1) \cos _{0}^{-1}(z)\right)}{\sin \left(\cos _{10}^{-1}(z)\right)} . z \in \Omega .
$$

For $ı=0.1 .2, \ldots k-1$. let

$$
L_{\imath}(z)=\cos \left(\frac{1}{k} \cos _{\imath}^{-1}(z)\right), z \in \Omega \text {. }
$$

Then $T_{k}\left(L_{\imath}(z)\right)=z, z \in \Omega$, so that $L_{\imath}$ is a branch in $\Omega$ of the multi-valued function $T_{k}^{-1}(z)$. The following results will be needed in the sequel. In all of them we assume $k \geq 2$.

LEMMA 1. For each $z$ in $\Omega$ and each polynomial $P(x)$ in $\mathbb{C}[x]$ of degree $m \geq 0$. $\sum_{z=0}^{h-1} P\left(L_{\imath}(z)\right)$ is a polynomial in $z$ of degree at most $[\mathrm{m} / \mathrm{k}]$, where $[a]$ denotes the largest integer $\leq u$.

PROOF. Partial fractions and the geometric series give for $|x|$ large that

$$
\frac{U_{k-1}(x)}{T_{k}(x)-z}=\sum_{\imath=0}^{k-1} \frac{1 / k}{x-L_{\imath}(z)}=\sum_{n=0}^{\infty}\left\{\frac{1}{k} \sum_{\imath=0}^{k-1} L_{\imath}^{n}(z)\right\} x^{-n-1} .
$$

For $|x|$ large we also have

$$
\frac{U_{k-1}(x)}{T_{k}(x)-z}=\sum_{j=0}^{\infty} \frac{U_{k-1}(x)}{T_{k}^{j+1}(x)} z^{\jmath}
$$

and long division gives

$$
\frac{U_{k-1}(x)}{T_{k}^{j+1}(x)}=\sum_{n=\jmath k}^{\infty} \frac{a_{n} j}{x^{n+1}}
$$

Hence

$$
\frac{U_{k-1}(x)}{T_{k}(x)-z}=\sum_{n=0}^{\infty} \frac{p_{n}(z)}{x^{n+1}}
$$

where $p_{n}(z)=\sum_{\jmath=0}^{[n / k]} a_{n j} z^{\jmath}$. Thus $\sum_{\imath=0}^{k-1} L_{\imath}^{n}(z)=k p_{n}(z)$, and the assertion follows.

Now let $-1<\xi_{1}<\xi_{2}<\cdots<\xi_{k-1}<1$ be the roots of $U_{k-1}(x)$. For $j=0,1, \ldots, k-1$, let

$$
A_{\jmath}=\sum_{T_{k}\left(\xi_{\imath}\right)=1} \frac{U_{\jmath}\left(\xi_{\imath}\right)}{U_{k-1}^{\prime}\left(\xi_{\imath}\right)}, \quad B_{\jmath}=\sum_{T_{k}\left(\xi_{\imath}\right)=-1} \frac{U_{\jmath}\left(\xi_{\imath}\right)}{U_{k-1}^{\prime}\left(\xi_{\imath}\right)} .
$$

Then

LEMMA 2. For $j=0,1,2, \ldots, k-1$,

$$
A \jmath+B,=0, \jmath \neq k-2 ; A_{k-2}+B_{k-2}=\frac{1}{2} .
$$

PROOF. Partial fractions decomposition gives for large $|x|$ that

$$
\frac{U_{j}(x)}{U_{k-1}(x)}=\sum_{j=1}^{k-1} \frac{U_{\jmath}\left(\xi_{\imath}\right)}{U_{k-1}^{\prime}\left(\xi_{\imath}\right)} \cdot \frac{1}{x-\xi_{\imath}}=\sum_{n=0}^{\infty}\left\{\sum_{\imath=1}^{k-1} \frac{U_{j}\left(\xi_{\imath}\right)}{U_{k-1}^{\prime}\left(\xi_{\imath}\right)} \xi_{\imath}^{n}\right\} \frac{1}{x^{n+1}}
$$


Since the coefficient of $1 / x$ in the long division of $U_{j}(x)$ by $U_{k-1}(x)$ is 0 if $J \neq k-2$ and $1 / 2$ if $\jmath=k-2$, the assertion follows.

LEMMA 3. For $\mathrm{J}=0,1,2, \ldots, k-1$,

$$
A_{k-\jmath-2}=-A_{\jmath} . \quad B_{k-\jmath-2}=B_{\jmath} .
$$

PROOF. This is a consequence of the trigonometric identity $U_{k-\jmath-2}(x)=U_{k-1}(x) T_{\jmath+1}(x)$ $-U_{\jmath}(x) T_{k}(x)$.

LEMMA 4. For $z \in \Omega$ and $\jmath=0,1,2, \ldots, k-1$,

$$
\frac{1}{k} \sum_{\imath=0}^{k-1} \frac{U_{\jmath}\left(L_{\imath}(z)\right)}{U_{k-1}^{2}\left(L_{\imath}(z)\right)}=\frac{A \jmath}{z-1}+\frac{B_{\jmath}}{z+1}
$$

PROOF. Partial fraction decomposition gives for $|x|$ large that

$$
\begin{aligned}
\frac{U_{\jmath}(x)}{U_{k-1}(x)\left(T_{k}(x)-z\right)} & =\sum_{\imath=1}^{k-1} \frac{U_{\jmath}\left(\xi_{\imath}\right)}{U_{k-1}^{\prime}\left(\xi_{\imath}\right)\left(T_{k}\left(\xi_{\imath}\right)-z\right)\left(x-\xi_{\imath}\right)}+\sum_{\imath=0}^{k-1} \frac{U_{\jmath}\left(L_{\imath}(z)\right)}{k U_{k-1}^{2}\left(L_{\imath}(z)\right)\left(x-L_{\imath}(z)\right)} \\
& =\sum_{n=0}^{\infty}\left\{\sum_{\imath=1}^{k-1} \frac{U_{\jmath}\left(\xi_{\imath}\right) \xi_{\imath}^{n}}{U_{k-1}^{\prime}\left(\xi_{\imath}\right)\left(T_{k}\left(\xi_{\imath}\right)-z\right)}+\sum_{\imath=0}^{k-1} \frac{U_{\jmath}\left(L_{\imath}(z)\right) L_{\imath}^{n}(z)}{k U_{k-1}^{2}\left(L_{\imath}(z)\right)}\right\} \frac{1}{x^{n+1}} .
\end{aligned}
$$

Since $k \geq 2$, long division on the left hand side of the above equality gives 0 for the coefficient of $1 / x$, and (2.14) follows at once.

The relationships

$$
L_{\imath}^{\prime}(z)=\frac{1}{k U_{k-1}\left(L_{\imath}(z)\right)},\left(T_{\jmath}\left(L_{\imath}(z)\right)\right)^{\prime}=\frac{j U_{\jmath-1}\left(L_{\imath}(z)\right)}{k U_{k-1}\left(L_{\imath}(z)\right)}, z \in \Omega,
$$

and the trigonometric identity

$$
U_{\jmath-\imath}(x)=U_{\imath}(x) U_{\jmath}(x)-U_{\imath-1}(x) U_{\jmath+1}(x), j \geq i \geq 0,
$$

will be also needed.

Now let $\gamma$ be the positively oriented ellipse

$$
\frac{x^{2}}{a^{2}}+\frac{y^{2}}{b^{2}}=1, \quad a^{2}-b^{2}=1, \quad a>1,
$$

so that, for some $y_{0}>0, a=\cosh y_{0}, b=\sinh y_{0}$. Observe that $[-1,1]$ is in the interior of $\gamma$. For each $i=1,2, \ldots, k-1$, let $\gamma_{\imath}=L_{\imath} \circ \gamma$ be the $i^{t h}$-lifting of $\gamma$ through $T_{k}$. The $\gamma_{\imath}$ 's piece together onto the ellipse $\tilde{\gamma}$ determined by

$$
\frac{x^{2}}{A^{2}}+\frac{y^{2}}{B^{2}}=1
$$

where $A=\cosh y_{0} / k, B=\sinh y_{0} / k$. If $|z-1|>2$ for $z$ on $\gamma$, then $\left|T_{k}(z)-1\right|>2$ for $z$ on $\tilde{\gamma}$. This follows from $\left|T_{k}\left(L_{\imath}(z)\right)-1\right|=|z-1|$. If $f$ is continuous on $\widetilde{\gamma}$ then

$$
\int_{\gamma_{\mathrm{\imath}}} f(z) d z=\frac{1}{k} \int_{\gamma} \frac{f\left(L_{\imath}(z)\right)}{U_{k-1}\left(L_{\imath}(z)\right)} d z, \int_{\gamma} f(z) d z=\sum_{\imath=0}^{k-1} \int_{\gamma_{\mathrm{\imath}}} f(z) d z .
$$

For $f$ continuous on $\gamma$ we also have

$$
\int_{\tilde{\gamma}} f\left(T_{k}(z)\right) U_{k-1}(z) d z=\int_{\gamma} f(z) d z
$$


Since

$$
\int_{\gamma} \frac{d z}{z-1}=\sum_{\imath=0}^{k-1} \frac{1}{k} \int_{\gamma} \frac{d z}{U_{k-1}\left(L_{\imath}(z)\right)\left(L_{\imath}(z)-1\right)}=\int_{\gamma} \frac{d z}{z-1}=2 \pi \iota .
$$

$\tilde{\gamma}$ is simple and positively oriented. We call $\tilde{\gamma}$ the lifting of $\gamma$ through $T_{k}$. If $C$ is any positively oriented contour of $\left|T_{k}(z)-1\right|>2$ containing $[-1,1]$ nn its interior and $f$ is analytic on this set. then

$$
\int_{C} f(z) d z=\int_{\gamma} f(z) d z
$$

provided that $|z-1|>2$ on $\gamma$.

3. CONTOUR INTEGRAL REPRESENTATION OF $\mathcal{L}_{1}$.

We will write $p_{n}(x)$ instead of $p_{n}^{\lambda}(x)$ to denote the $n^{\text {th }}$ sieved ultraspherical polynomial of the first kind. Results in Al-Salam et al. [2], Charris and Ismail [5-7] or Charris et al. [8] yield

$$
\begin{aligned}
& U_{k-1}(x) p_{n k+\jmath}(x) \\
& =\frac{n !}{2^{n k+\jmath}(\lambda)_{n+1}}\left\{(n+1) U_{\jmath-1}(x) C_{n+1}^{\lambda}\left(T_{k}(z)\right)+(n+2 \lambda) U_{k-\jmath-1}(x) C_{n}^{\lambda}\left(T_{k}(x)\right)\right\}
\end{aligned}
$$

for $n \geq 0, \jmath=1,2, \ldots, k$. In particular, $p_{n k}(x)=\frac{n^{\prime}}{2^{n k}(\lambda)_{n}} C_{n}^{\lambda}\left(T_{k}(x)\right), n \geq 0$.

For $z$ in $\mathbb{C}$ such that $\left|T_{k}(z)-1\right|>2,(1.12)$ and results in the above references, which were established for the positive definite case, i.e., when (1.8) holds, suggest that

$$
\Phi(z)=U_{k-1}(z) X^{\lambda}\left(T_{k}(z)\right)=\frac{U_{k-1}(z)}{T_{k}(z)-1}{ }_{2} F_{1}\left(\begin{array}{cc|c}
1, & \lambda+\frac{1}{2} & \frac{2}{1-T_{k}(z)}
\end{array}\right)
$$

is the limit of the continued fraction of $\left\{p_{n}(x)\right\}$. That this is so follows from Theorem 3 and from the next theorem.

THEOREM 4. Let

$$
\mathcal{L}(P(x))=\frac{1}{2 \pi i} \int_{C} P(z) \Phi(z) d z
$$

where $C$ is a positively oriented contour of $\left\|T_{k}(z)-1\right\|>2$ containing $[-1,1]$ in its interior. Then $\mathcal{L}$ is the moment functional $\mathcal{L}_{1}$ of $\left\{p_{n}(x)\right\}$.

PROOF. We may assume that $C$ is the lifting through $T_{k}$ of an ellipse $\gamma$ in $|z-1|>2$, as in $(2.17)$, containing $[-1,1]$ in its interior. That $\mathcal{L}(1)=1$ follows from

$$
\int_{C} \Phi(z) d z=\int_{C} U_{k-1}(z) X^{\lambda}\left(T_{k}(z)\right) d z=\int_{\gamma} X^{\lambda}(z) d z
$$

Now, from (2.15) and (2.19),

$$
\begin{aligned}
\int_{C} U_{\jmath-1}(z) C_{n+1}^{\lambda}\left(T_{k}(z)\right) X^{\lambda}\left(T_{k}(z)\right) d z & =\int_{\gamma} \sum_{\imath=0}^{k-1} \frac{U_{\jmath-1}\left(L_{\imath}(z)\right)}{k U_{k-1}\left(L_{\imath}(z)\right)} C_{n+1}^{\lambda}(z) X^{\lambda}(z) d z \\
& =\frac{1}{j} \int_{\gamma}\left(\sum_{\imath=0}^{k-1} T_{\jmath}\left(L_{\imath}(z)\right)\right)^{\prime} C_{n+1}^{\lambda}(z) X^{\lambda}(z) d z,
\end{aligned}
$$

so that, in view of Lemma 1 , the integral vanishes if $j=1,2, \ldots, k-1$. If $j=k$ it also vanishes, as it reduces to $2 \pi i \mathcal{L}_{0}\left(C_{n+1}^{\lambda}(x)\right)$. Noting that $k-j-1<k$ for $j=1,2, \ldots, k$, we also have that

$$
\int_{C} U_{k-\jmath-1}(z) C_{n}^{\lambda}\left(T_{k}(z)\right) X^{\lambda}\left(T_{k}(z)\right) d z=0, j=1,2, \ldots, k,
$$


and taking into account (3.1) we conclude that $\mathcal{L}\left(p_{n k+\jmath}(x)\right)=0, n \geq 0, \jmath=1,2, \ldots, k$.

4. CONTOUR INTEGRAL REPRESENTATION OF $\mathcal{L}_{2}$.

We write $q_{n}(x)$ to denote $q_{n}^{\lambda}(x)$. Results established in Charris and Ismail [5-7] or Charris et al. [8] show that

$$
q_{n k+\jmath}(x)=\frac{1}{2^{n k+\jmath}}\left\{U_{\jmath}(x) C_{n}^{\lambda+1}\left(T_{k}(x)\right)+U_{k-\jmath-2}(x) C_{n-1}^{\lambda+1}\left(T_{k}(x)\right)\right\},
$$

$n \geq 0, \jmath=0,1 \ldots, k-1$. In particular,

$$
q_{(n+1) k-1}(x)=\frac{n !}{2^{(n+1) k-1}(\lambda+1)_{n}} U_{k-1}(x) C_{n}^{\lambda+1}\left(T_{k}(x)\right), n \geq 0 .
$$

Hence, for $|z-1|>2$,

$$
Y^{\lambda}(z):=X^{\lambda+1}(z)=\frac{1}{z+1}{ }_{2} F_{1}\left(1, \lambda+\frac{3}{2} 2 \lambda+3 \mid \frac{2}{z \pm 1}\right)
$$

is the limit of the continued fraction of $\left\{C_{n}^{\lambda+1}(x)\right\}$ and, for $\left|T_{k}(z)-1\right|>2, \Psi(z)$, given by

$$
\Psi(z)=2\left\{\frac{U_{k-2}(z)}{U_{k-1}(z)}+\frac{1+2 \lambda}{2(1+\lambda)} \frac{Y^{\lambda}\left(T_{k}(z)\right)}{U_{k-1}(z)}\right\}
$$

will be that of $\left\{q_{n}(x)\right\}$. This will follow from Theorem 5 below. Relation (4.4) is suggested by results in the above references for the positive definite case. To simplify the notation, let

$$
Q_{n k+\jmath}(x)=\frac{2^{n k+\jmath}(\lambda+1)_{n}}{n !} q_{n k+\jmath}(x), n \geq 0, j=0,1,2, \ldots, k-1,
$$

and define

$$
\mathcal{L}(P(x))=\frac{1}{2 \pi i} \int_{C} P(z) \Psi(z) d z, P(x) \in \mathbb{C}[x],
$$

where $C$ is a positively oriented closed contour of $\left|T_{k}(z)-1\right|>2$ containing $[-1,1]$ in its interior. We claim that

THEOREM 5. If $\mathcal{L}$ is given by (4.5) then $\mathcal{L}(1)=1$ and $\mathcal{L}\left(q_{n}(x)\right)=0$ if $n \geq 1$. Hence, if $2 \lambda \neq 0$ and is not a negative integer, $\mathcal{L}$ is the orthogonality functional $\mathcal{L}_{2}$ of $\left\{q_{n}(x)\right\}$.

PROOF. For each $n \geq 0$ and $j=0,1, \ldots, k-1$, let

$$
I(n, j)=\frac{1}{2 \pi i} \int_{C} U_{j}(z) C_{n}^{\lambda+1}\left(T_{k}(z)\right) \Psi(z) d z .
$$

As before, we may assume that $C$ is the lifting through $T_{k}$ of an ellipse $\gamma$ in $|z-1|>2$ as in (2.17). We have

$$
\mathcal{L}\left(Q_{n k+\jmath}(x)\right)=I(n, j)+I(n-1, k-j-2) .
$$

Now, for $n \geq 1$ and $0 \leq j \leq k-2$, we obtain, from (2.19) and (4.4), that

$$
\begin{aligned}
I(n, j)= & \frac{1}{\pi i} \int_{\gamma}\left\{\sum_{\imath=0}^{k-1} \frac{U_{k-2}\left(L_{\imath}(z)\right) U_{\jmath}\left(L_{\imath}(z)\right)}{k U_{k-1}^{2}\left(L_{\imath}(z)\right)}\right\} C_{n}^{\lambda+1}(z) d z \\
& +\frac{2 \lambda+1}{2(\lambda+1)} \cdot \frac{1}{\pi i} \int_{\gamma}\left\{\sum_{\imath=0}^{k-1} \frac{U_{\jmath}\left(L_{\imath}(z)\right)}{k U_{k-1}^{2}\left(L_{\imath}(z)\right)}\right\} C_{n}^{\lambda+1}(z) Y^{\lambda}(z) d z .
\end{aligned}
$$

Using (2.16) and observing that

$$
j \cdot \sum_{\imath=0}^{k-1} \frac{U_{\jmath-1}\left(L_{\imath}(z)\right)}{k U_{k-1}\left(L_{\imath}(z)\right)}=\sum_{\imath=0}^{k-1}\left(T_{\jmath}\left(L_{\imath}(z)\right)\right)^{\prime}=0
$$


as follows from (2.15) and Lemma 1. we obtain. by means of Lemma 4, that

$$
\begin{aligned}
I(n . \jmath)=\frac{1}{\pi \imath}\left\{A_{h-\jmath-2} \int_{\gamma} \frac{C_{n}^{\lambda+1}(z)}{z-1} d z+B_{h-\jmath-2} \int_{\gamma} \frac{C_{n}^{\lambda+1}(z)}{z+1} d z\right. \\
\left.\quad+A_{\jmath} \frac{2 \lambda+1}{2(\lambda+1)} \int_{\gamma} \frac{C_{n}^{\lambda+1}(z)}{z-1} Y^{-\lambda}(z) d z+B_{\jmath} \frac{2 \lambda+1}{2(\lambda+1)} \int_{\gamma} \frac{C_{n}^{\lambda+1}(z)}{z+1} Y^{-\lambda}(z) d z\right\}
\end{aligned}
$$

Cauchy`s formula and $(1.23),(1.25),(1.26)$ then imply that

$$
I(n, \jmath)=2\left\{\left(A_{k-\jmath-2}+(-1)^{n} B_{k-\jmath-2}\right) C_{n}^{\lambda}(1)+\left(A_{\jmath}+(-1)^{n+1} B_{\jmath}\right)(2 \lambda+1) C_{n-1}^{\lambda+1}(1 ; 1)\right\}
$$

which, in view of (2.13) and the obvious relationship $(2 \lambda+1) C_{n-1}^{\lambda+1}(1 ; 1)=C_{n}^{\lambda+1}(1)-1$, reduces to $I(n, \jmath)=-2\left(A_{\jmath}+(-1)^{n+1} B_{\jmath}\right)$.

Also $I(n-1, k-j-2)=-2\left(A_{k-\jmath-2}+(-1)^{n} B_{k-\jmath-2}\right)$, so that, using (2.13) again, we obtain

$$
\mathcal{L}\left(Q_{n k+\jmath}(x)\right)=0, n \geq 1, \jmath=0,1,2, \ldots, k-2 .
$$

As for the case $n \geq 1, \jmath=k-1$, , i.e., of $Q_{(n+1) k-1}(x)$, we obtain, from (4.2), that

$$
\begin{aligned}
& \mathcal{L}\left(Q_{(n+1) k-1}(x)\right)= \\
& \frac{1}{\pi i} \int_{C} U_{k-2}(z) C_{n}^{\lambda+1}\left(T_{k}(z)\right) d z+\frac{1}{\pi i} \frac{2 \lambda+1}{2(\lambda+1)} \int_{\gamma}\left(\sum_{\imath=0}^{k-1} L_{\imath}(z)\right)^{\prime} C_{n}^{\lambda+1}(z) Y^{\lambda}(z) d z
\end{aligned}
$$

which, in view of Lemma 1 and the analiticity of $U_{k-2}(z) C_{n}^{\lambda+1}\left(T_{k}(z)\right)$, also vanishes. Since finally $I(0, j)=2\left(A_{k-\jmath-2}+B_{k-\jmath-2}\right)=0$ if $j \neq 0$ and $I(0,0)=1$, we conclude that $\mathcal{L}(1)=1$ and $\mathcal{L}\left(q_{n}(x)\right)=0$ if $n>0$.

REMARK 1. With

$$
\widehat{\Psi}(z)=\frac{2 \lambda+1}{\lambda+1} \frac{Y^{\lambda}\left(T_{k}(z)\right)}{U_{k-1}(z)}, \quad\left|T_{k}(z)-1\right|>2,
$$

we obtain the alternative representation

$$
\mathcal{L}_{2}(P(z))=2 \sum_{\imath=1}^{k-1} \frac{U_{k-2}\left(\xi_{\imath}\right)}{U_{k-2}^{\prime}\left(\xi_{\imath}\right)} P\left(\xi_{\imath}\right)+\frac{1}{2 \pi i} \int_{C} P(z) \widehat{\Psi}(z) d z
$$

where $\xi_{1}<\xi_{2}<\cdots<\xi_{k-1}$ are the roots of $U_{k-1}(x)$.

5. DISTRIBUTIONAL REPRESENTATIONS OF $\mathcal{L}_{1}$ AND $\mathcal{L}_{2}$.

From the contour integral representations of $\mathcal{L}_{1}$ and $\mathcal{L}_{2}$ it is easy to derive distributional representations of these functionals.

Let, as before, $-1<\xi_{1}<\xi_{2}<\cdots<\xi_{k-1}<1$ be the roots of $U_{k-1}(x)$, and let $\xi_{0}=-1, \xi_{k}=$ 1. Then $\xi_{0}, \xi_{k}$ are simple roots of $T_{k}^{2}(x)-1$, while for $i=1,2, \ldots, k-1, \xi_{2}$ is a double root. Hence, for $m \geq 0$ an integer and $P(x) \in \mathbb{C}[x]$,

$$
\frac{P(x)}{\left(1-T_{k}^{2}(x)\right)^{m}}=\sum_{\imath=0}^{k} \sum_{\jmath=1}^{l_{\imath}} \frac{\alpha_{\imath \jmath}}{\left(x-\xi_{\imath}\right)^{\jmath}}+R(x) ; \quad \alpha_{\imath \jmath}=\frac{1}{\left(l_{\imath}-j\right) !} \frac{d^{l_{\imath}-\jmath}}{d x^{l_{\imath}-\jmath}}\left[\frac{\left(x-\xi_{\imath}\right)^{l_{\imath}}}{\left(1-T_{k}^{2}(x)\right)^{m}} P(x)\right]\left(\xi_{\imath}\right),
$$

with $l_{0}=l_{k}=m, l_{\imath}=2 m$ for $i=1,2, \ldots, k-1$, and $R(x)$, a polynomial.

Since

$$
\mathcal{L}_{1}(P(x))=\frac{1}{2 \pi i} \frac{\Gamma(\lambda+1)}{\sqrt{\pi} \Gamma(\lambda+1 / 2)} \int_{C} P(z) X^{\lambda-1 / 2, \lambda-1 / 2}\left(T_{k}(z)\right) U_{k-1}(z) d z
$$


follows from(1.17), then, provided $2 \lambda$ is not an integer $\leq 0$,

$$
\begin{aligned}
\mathcal{L}_{1}(P(x))= & \sum_{\imath=0}^{k} \sum_{\jmath=0}^{l_{\imath}-1} A_{\imath \jmath} \frac{1}{\jmath !} \frac{d^{\jmath}}{d x^{\jmath}}\left[\frac{\left(x-\xi_{\imath}\right)^{l_{1}}}{\left.1-T_{k}^{2}(x)\right)^{m}} P(x)\right]\left(\xi_{\imath}\right)+ \\
& +\frac{1}{2 \pi \imath} \frac{\Gamma(\lambda+1)}{\sqrt{\pi} \Gamma(\lambda+1 / 2)} \int_{C} R(z) U_{k-1}(z) X^{\lambda+m-1 / 2, \lambda+m-1 / 2}\left(T_{k}(z)\right) d z
\end{aligned}
$$

where

$$
A_{\imath \jmath}=\frac{1}{2 \pi \imath} \int_{C} \frac{U_{k-1}(z)\left(1-T_{k}^{2}(z)\right)^{m}}{\left(z-\xi_{\imath}\right)^{l_{i}-\jmath}} X^{\lambda}\left(T_{k}(z)\right) d z
$$

for $\imath=0,1,2, \ldots, k$ and $\jmath=0,1, \ldots, l_{\imath}-1$. Observe that $A_{\imath}$ is independent of $P(x)$, and also of $C$, as long as $C$ is contained in $\left|T_{k}(z)-1\right|>2$.

Now assume $C$ is the lifting through $T_{k}(x)$ of an ellipse (2.17), $\gamma$, in $|z-1|>2$. Then

$$
\begin{aligned}
& \frac{1}{2 \pi i} \int_{C} R(z) U_{k-1}(z) X^{\lambda+m-1 / 2, \lambda+m-1 / 2}\left(T_{k}(z)\right) d z \\
& =\frac{1}{2 \pi i k} \sum_{i=0}^{k-1} \int_{\gamma} R\left(L_{\imath}(z)\right) X^{\lambda+m-1 / 2, \lambda+m-1 / 2}(z) d z \\
& =\frac{1}{k} \sum_{i=0}^{k-1} \int_{-1}^{1} R\left(L_{\imath}(z)\right)\left(1-x^{2}\right)^{\lambda+m-1 / 2} d x \\
& =\sum_{i=0}^{k-1} \int_{\xi_{1}}^{\xi_{1+1}} R(x)\left(1-T_{k}^{2}(x)\right)^{\lambda+m-1 / 2}\left|U_{k-1}(x)\right| d x \\
& =\int_{-1}^{1} R(x)\left|U_{k-1}(x)\right|^{2(\lambda+m)}\left(1-x^{2}\right)^{\lambda+m-1 / 2} d x .
\end{aligned}
$$

Hence

THEOREM 6. If $2 \lambda \neq 0$ is not a negative integer and $\lambda>-m-1 / 2$, where $m \geq 0$ is an integer, the functional $\mathcal{L}_{1}$ has the distributional representation $\mathcal{L}_{1}=T_{11}+T_{12}$, where, for any test function $\varphi$,

$$
T_{11}(\varphi)=\sum_{\imath=0}^{k} \sum_{\jmath=0}^{l_{\mathrm{s}}-1} A_{\imath} \frac{1}{j !} \frac{d^{\jmath}}{d x^{j}}\left[\frac{\left(x-\xi_{\imath}\right)^{l_{\mathrm{l}}}}{\left(1-T_{k}^{2}(x)\right)^{m}} \varphi(x)\right]\left(\xi_{\imath}\right)
$$

with $A_{i j}$ given by (5.2), $l_{0}=l_{k}=m, l_{i}=2 m$ for $i=1,2, \ldots, k-1$, and

$$
T_{12}(\varphi)=\frac{\Gamma(\lambda+1)}{\sqrt{\pi} \Gamma(\lambda+1 / 2)} \int_{-1}^{1} \varphi_{m}(x)\left|U_{k-1}(x)\right|^{2(\lambda+m)}\left(1-x^{2}\right)^{\lambda+m-1 / 2} d x
$$

with

$$
\varphi_{m}(x)=\frac{\varphi(x)}{\left(1-T_{k}^{2}(x)\right)^{m}}-\sum_{\imath=0}^{k} \sum_{j=0}^{l_{i}-1} \frac{1}{j !\left(x-\xi_{\imath}\right)^{l_{i}-1}} \frac{d^{j}}{d x^{j}}\left[\frac{\left(x-\xi_{z}\right)^{l_{i}}}{\left(1-T_{k}^{2}(x)\right)^{m}} \varphi(x)\right]\left(\xi_{\imath}\right)
$$

Both $T_{11}$ and $T_{12}$ are compactly supported on the real line and can act on polynomials. As for $\mathcal{L}_{2}$, observe that, from (4.4),

$$
\begin{aligned}
\Psi(z)= & 2 \frac{U_{k-2}(z)}{U_{k-1}(z)}+ \\
& +\frac{\Gamma(\lambda+1)}{\sqrt{\pi} \Gamma(\lambda+1 / 2)}\left[\frac{1}{1-T_{k}(z)}+\frac{1}{1+T_{k}(z)}\right] \cdot\left(1-z^{2}\right) U_{k-1}(z) X^{\lambda+1 / 2, \lambda+1 / 2}\left(T_{k}(z)\right),
\end{aligned}
$$


and simple calculations with the series involved show that

$$
\begin{aligned}
& \Psi(z)= \\
& 2 z+\frac{\Gamma(\lambda+1)}{\sqrt{\pi} \Gamma(\lambda+1 / 2)}\left[X^{\lambda-1 / 2, \lambda+1 / 2}\left(T_{k}(z)\right)+X^{\lambda+1 / 2, \lambda-1 / 2}\left(T_{k}(z)\right)\right]\left(1-z^{2}\right) U_{k-1}(z) .
\end{aligned}
$$

Hence

THEOREM 7. If $2 \lambda \neq 0$ is not a negative integer and $\lambda>-m-1 / 2$, where $m \geq 0$ is an integer, the functional $\mathcal{L}_{2}$ can be represented in the form $\mathcal{L}_{2}=T_{21}+T_{22}$, where, for any test function $\varphi$,

$$
T_{21}(\varphi)=\sum_{\imath=0}^{k} \sum_{\jmath=0}^{l_{\imath}-1} B_{\imath} \frac{1}{j !} \frac{d^{\jmath}}{d x^{\jmath}}\left[\frac{\left(x-\xi_{\imath}\right)^{l_{\imath}}}{\left(1-T_{k}^{2}(x)\right)^{m}} \varphi(x)\right]\left(\xi_{\imath}\right)
$$

with

$$
\begin{aligned}
B_{\imath \jmath}= & \frac{\Gamma(\lambda+1)}{\sqrt{\pi} \Gamma(\lambda+1 / 2)} \\
& \cdot \frac{1}{2 \pi i} \int_{C} \frac{\left(1-z^{2}\right)^{m+1} U_{k-1}^{2 m+1}(z)}{\left(z-\xi_{\imath}\right)^{l_{\imath}-\jmath}} \cdot\left\{X^{\lambda-1 / 2, \lambda+1 / 2}\left(T_{k}(z)\right)+X^{\lambda+1 / 2, \lambda-1 / 2}\left(T_{k}(z)\right)\right\} d z
\end{aligned}
$$

and $l_{\imath}, i=0,1, \ldots, k$, as in Theorem 6 , and where

$$
T_{22}(\varphi)=\frac{2 \Gamma(\lambda+1)}{\sqrt{\pi} \Gamma(\lambda+1 / 2)} \int_{-1}^{1} \varphi_{m}(x)\left|U_{k-1}(z)\right|^{2(\lambda+m)}\left(1-x^{2}\right)^{\lambda+m+1 / 2} d x
$$

with $\varphi_{m}(x)$ as in (5.5). Both distributions $T_{21}$ and $T_{22}$ have compact support on the real line and can act on polynomials.

REMARK 2. If $k=1$ is allowed in Theorem $6, T_{11}$ and $T_{12}$ reduce to $T_{01}$ and $T_{02}$, respectively.

REMARK 3. If $\lambda>-1 / 2$, i.e., if we can assume $m=0$ in Theorems 6 and 7 , then $T_{11}=T_{21}=0$ and $T_{12}, T_{22}$ reduce, respectively, to the orthogonality measures of the sieved ultraspherical polynomials of the first and second kinds (as given in Al-Salam et al. [2]).

REMARK 4. When $\lambda \leq-1 / 2$, so that $m>0, T_{01}, T_{11}$ and $T_{21}$ measure the contributions to the orthogonality of the points in $[-1,1]$ where $\left(1-x^{2}\right)^{\lambda-1 / 2},\left|U_{k-1}(x)\right|^{2 \lambda}\left(1-x^{2}\right)^{\lambda-1 / 2}$ and $\left|U_{k-1}(x)\right|^{2 \lambda}\left(1-x^{2}\right)^{\lambda+1 / 2}$ become infinite.

It seems rather difficult to obtain distributional representations of $\mathcal{L}_{1}$ and $\mathcal{L}_{2}$ by the procedures in Krall [14], Morton and Krall [15].

ACKNOWLEDGEMENT. The first author acknowledges partial support of CINDEC, U.N., and of the N.S.F. under grant INT 8803099. The second author acknowledges the MAZDA Foundation for support through a graduate fellowship. The authors thank Dr. Mourad E. H. Ismail for suggesting the subject of this work. The first author also thanks Dr. Ismail for invitation and support to stay at the University of South Florida during September of 1991, where some of the ideas in this paper were rounded up. 


\section{REFERENC'ES}

1 AHKIEZER.I $\therefore$ The Classlcal Moment Problem. Hafner. New York. N.Y., 1965

2 AL-SALAM.W. ALLAWAY. IV and ASKEY. R Sieved ultraspherical polvnomials. Trans. Amer. Miath Sor. 284 (1984). 3458.

3 ASKEY, R and IS.MAIL. M. E. H. Recurrence relations, continued fractions and orthogonal polynomials, Mem. Amer. Math. Soc. 300 (1984), $110 \mathrm{pp}$.

4 CHARRIS. J. A. and GOMEZ, L. A. Functional analysis, orthogonal polynomials and a theorem of Markor. Rer. Col. de Mat. 22 (1988), 72-128.

5. CHARRIS, J. A. and ISMAIL, M. E. H. Sieved orthogonal polynomials II: Sieved random walk polynomials. Canadian J. of Math $\underline{33}$ (1986), 397-415.

6. CHARRIS, J. A., and ISMAIL, M. E. H. Sieved orthogonal polynomials V: Sieved Pollaczek polynomials SIAM. J. on Math. Anal. 18 (1987), 1177-1218.

7. CHARRIS, J. A.., and ISMAIL, M. E. H. Sieved orthogonal polynomials VII: Generalized polynomial mappings, Trans. Amer. Math. Soc. 340 (1993), 71-93.

8. CHARRIS, J. A., ISMAIL, M. E. H. and MONSALVE, S. Sieved orthogonal polynomials X: General blocks of recurrence relations, Pacific J. of Math., 163 (1994), 237-267

9. CHIHARA, T. S. An Introduction to Orthogonal Polynomials, Gordon and Breach, New York, N.Y., 1978.

10. GERONIMO, J. and VAN ASSCHE, W. Orthogonal polynomials on several intervals via a polynomial mapping, Trans. Amer. Math. Soc. 294 (1986), 89-111.

11. ISMAIL, M. E. H., Sieved orthogonal polynomials I: Sieved Pollaczek analogues, SIAM J. on Math. Anal. 16 (1985), 1093-1113.

12. ISMAIL, M. E. H. Sieved orthogonal polynomials III: Orthogonality on several intervals, Trans. Amer. Math. Soc. 194 (1986), 89-111.

13. ISMAIL, M. E. H., MASSON, D. and RAHMAN M. Complex weight functions for classical orthogonal polynomials, Canadian J. of Math $\underline{43}$ (1991), 1294-1308.

14. KRALL, A. Orthogonal polynomials through moment functionals, $\underline{\text { SIAM }} \underline{\mathrm{J}}$. on Math. Anal. $\underline{9}(1978), 600-603$.

15. MORTON, R. and KRALL, A. Distributional weight functions for orthogonal polynomials, SIAM J. on Math. Anal. 9 (1978), 604-626.

16. RAINVILLE, E. D. Special Functions, Macmillan, New York, N.Y., 1960.

17. SHOHAT, J. A. and TAMARKIN, J. D. The Problem of Moments, Math. Surveys, Amer. Math. Soc., Providence, R.I., 1943.

18. STONE, M. Linear Transformations in Hilbert Space and their Applications to Analysis, Colloquium Publications, Amer. Math. Soc., Providence, R.I., 1934.

19. SZEGÖ, G. Orthogonal Polynomials, $4^{\text {th }}$ Edition, Colloquium Publications, Amer. Math. Soc., Providence, R.I., 1975.

20. WALL, H. S. Analytic Theory of Continued Fractions, Chelsea, New York, N.Y., 1973. 


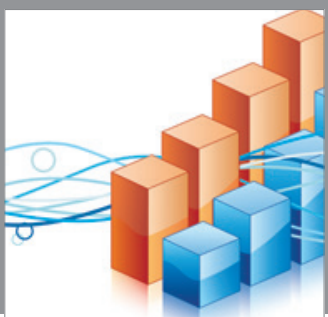

Advances in

Operations Research

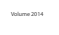

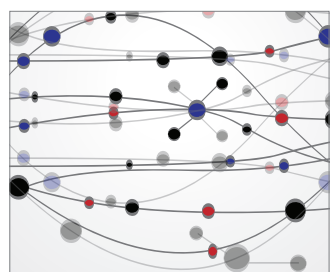

\section{The Scientific} World Journal
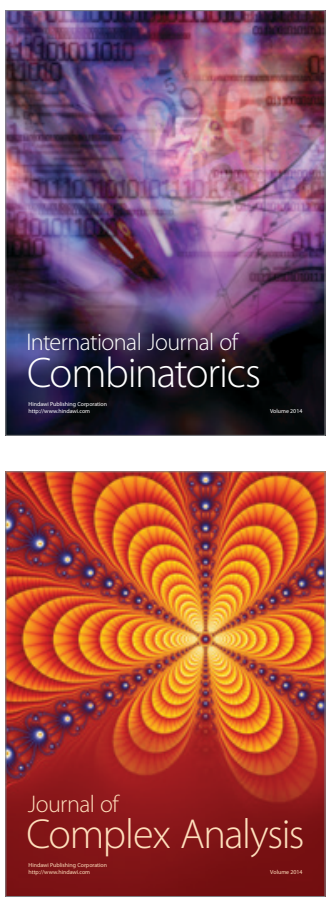

International Journal of

Mathematics and

Mathematical

Sciences
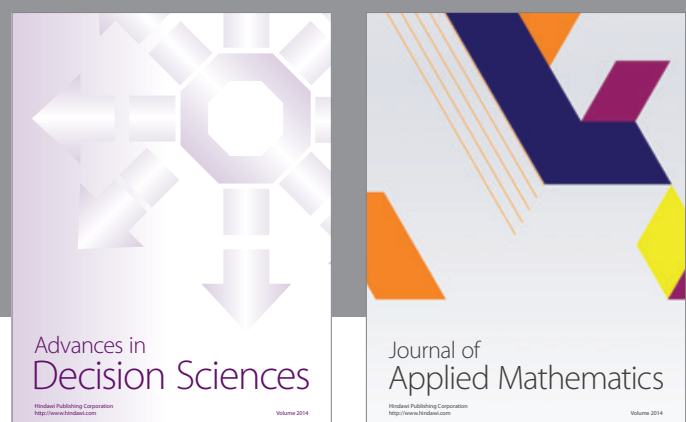

Journal of

Applied Mathematics
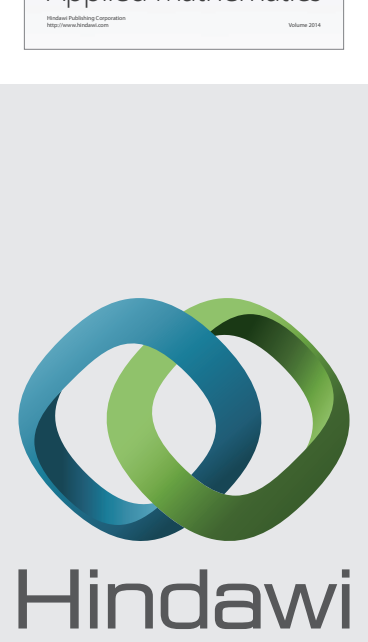

Submit your manuscripts at http://www.hindawi.com
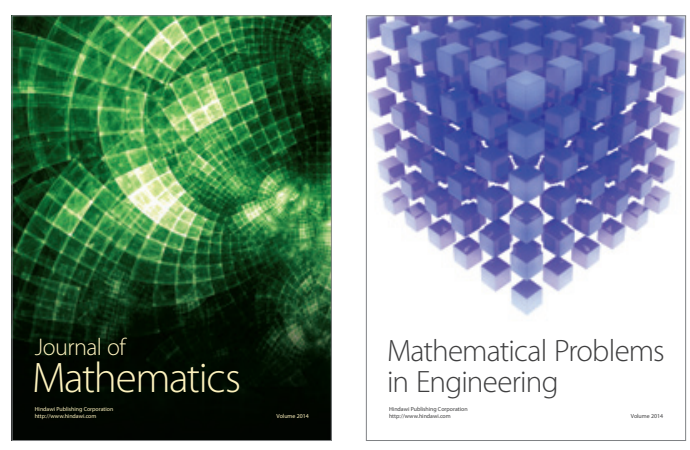

Mathematical Problems in Engineering
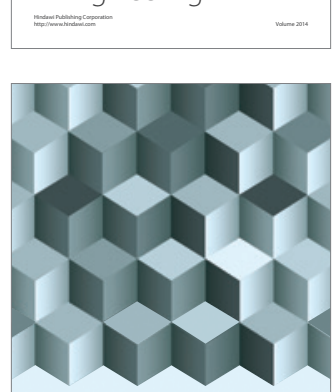

Journal of

Function Spaces
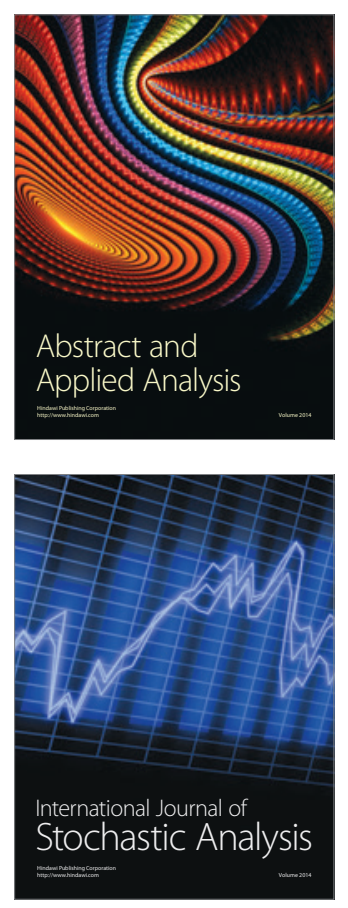

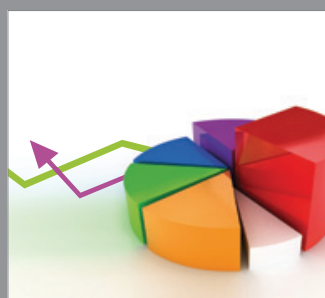

ournal of

Probability and Statistics

Promensencen
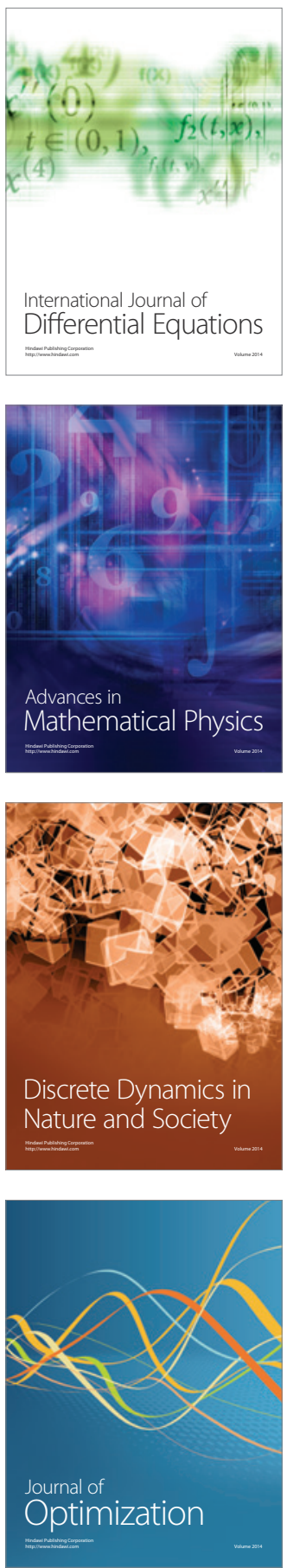